\title{
Dynamique sédimentaire lacustre de marge glaciaire. Le paléolac de Llestui (Noguera Ribagorçana - versant sud des Pyrénées).
} Jean-Manuel Vilaplana, Jaume Bordonau

\section{Résumé}

L'étude sédimentologique du complexe sédimentaire glaciaire de Llestui (Vallée de Llauset) formé du dernier cycle glaciaire, nous permet de reconnaître les différents processus sédimentaires du milieu lacustre juxtaglaciaire. En conséquence, on voit que la sédimentation glaciolacustre fine et rythmique présente des interruptions dues aux apports de flow-tills et de dépôts fluvio-torrentiels, intercalés dans les argiles laminées, souvent avec des contacts érosifs.

\begin{abstract}
The sedimentological study of the ice-lateral till complex of Llestui (Llauset Valley), formed during the last glacial cycle, allows us to recognize the several sedimentary processes in the ice-lateral lacustrine environment. Thus, the rhythmic glaciolacustrine sedimentation is interrupted by common flow-tills and fluvio-torrentiai deposits with erosional and interbedded contacts.
\end{abstract}

\section{Citer ce document / Cite this document :}

Vilaplana Jean-Manuel, Bordonau Jaume. Dynamique sédimentaire lacustre de marge glaciaire. Le paléolac de Llestui (Noguera Ribagorçana - versant sud des Pyrénées).. In: Bulletin de l'Association française pour l'étude du quaternaire, vol. 26, $\mathrm{n}^{\circ} 4,1989$. pp. 219-224.

doi : 10.3406/quate.1989.1911

http://www.persee.fr/doc/quate_0004-5500_1989_num_26_4_1911

Document généré le 30/09/2015 


\title{
DYNAMIQUE SÉDIMENTAIRE LACUSTRE DE MARGE GLACIAIRE : LE PALÉOLAC DE LLESTUI (NOGUERA RIBAGORÇANA - VERSANT SUD DES PYRÉNÉES)
}

\author{
par Joan Manuel VILAPLANA* et Jaume BORDONAU*
}

\begin{abstract}
RÉSUMÉ
L'étude sédimentologique du complexe sédimentaire glaciaire de Llestui (Vallée de Llauset) formé du dernier cycle glaciaire, nous permet de reconnaître les différents processus sédimentaires du milieu lacustre juxtaglaciaire. En consequence, on voit que la sédimentation glaciolacustre fine et rythmique presente des interruptions dues aux apports de flow-tills et de dépóts fluvio-torrentiels, intercalés dans les argiles laminées, souvent avec des contacts érosifs.
\end{abstract}

Mots-clés : Lac juxtaglaciaire, sédimentation glaciolacustre, diamicton, rythmites, Pyrénées.

\section{ABSTRACT}

ICE-MARGINAL LACUSTRINE SEDIMENTATION : THE LLESTUI PALEOLAKE (NOGUERA RIBAGORÇANA BASIN, CENTRAL SOUTHERN PYRENEES).

The sedimentological study of the ice-lateral till complex of Llestui (Llauset Valley), formed during the last glacial cycle, allows us to recognize the several sedimentary processes in the ice-lateral lacustrine environment. Thus, the rhythmic glaciolacustrine sedimentation is interrupted by common flow-tills and fluvio-torrential deposits with erosional and interbedded contacts.

Key-words : Ice-lateral lake, glaciolacustrine sedimentation, diamicton, rhythmites, Pyrenees.

\section{INTRODUCTION}

L'étude des sédiments glaciolacustres est importante pour faire une bonne reconstruction de la dynamique glaciaire pyrénéenne. C'est dans les petits lacs de marge glaciaire que l'on peut trouver la plupart de l'information stratigraphique. Un des meilleurs exemples de ce type de palélacs, que nous avons étudiés dans le versant sud des Pyrénées Centrales, est Llestui $(1600 \mathrm{~m})$ dans le haut bassin de la Noguera Ribagorçana (Vilaplana, 1983 a, b; Serrat et al., 1983), (fig. 1).

Ce palélac recevait les eaux d'un petit bassin versant constitue par des schistes graphiteux du Silurien et par des schistes calcaires du Dévonien.

La cartographie des faciès sédimentaires de cet environnement (Vilaplana, 1983a) nous a permis de faire une estimation des paramètres géométriques du lac. La cuvette lacustre, d'à peu près 8 ha, avait une longueur de l'ordre de 200 à $300 \mathrm{~m}$, et une largeur qui allait de 300 a $400 \mathrm{~m}$ (voir fig. 5).

* Departament de Geologia Dinàmica, Geofísica i Paleontologia. Universitat de Barcelona. Zona Universitària de Pedralbes, 08028 - BARCELONA. Espagne.

Contribution au colloque AFEQ "Sédiments lacustres laminés et environnement glaciaire actuel et passé". Toulouse 3 mai 1989 (organisé par G. Jalut et J. Hubschman). 


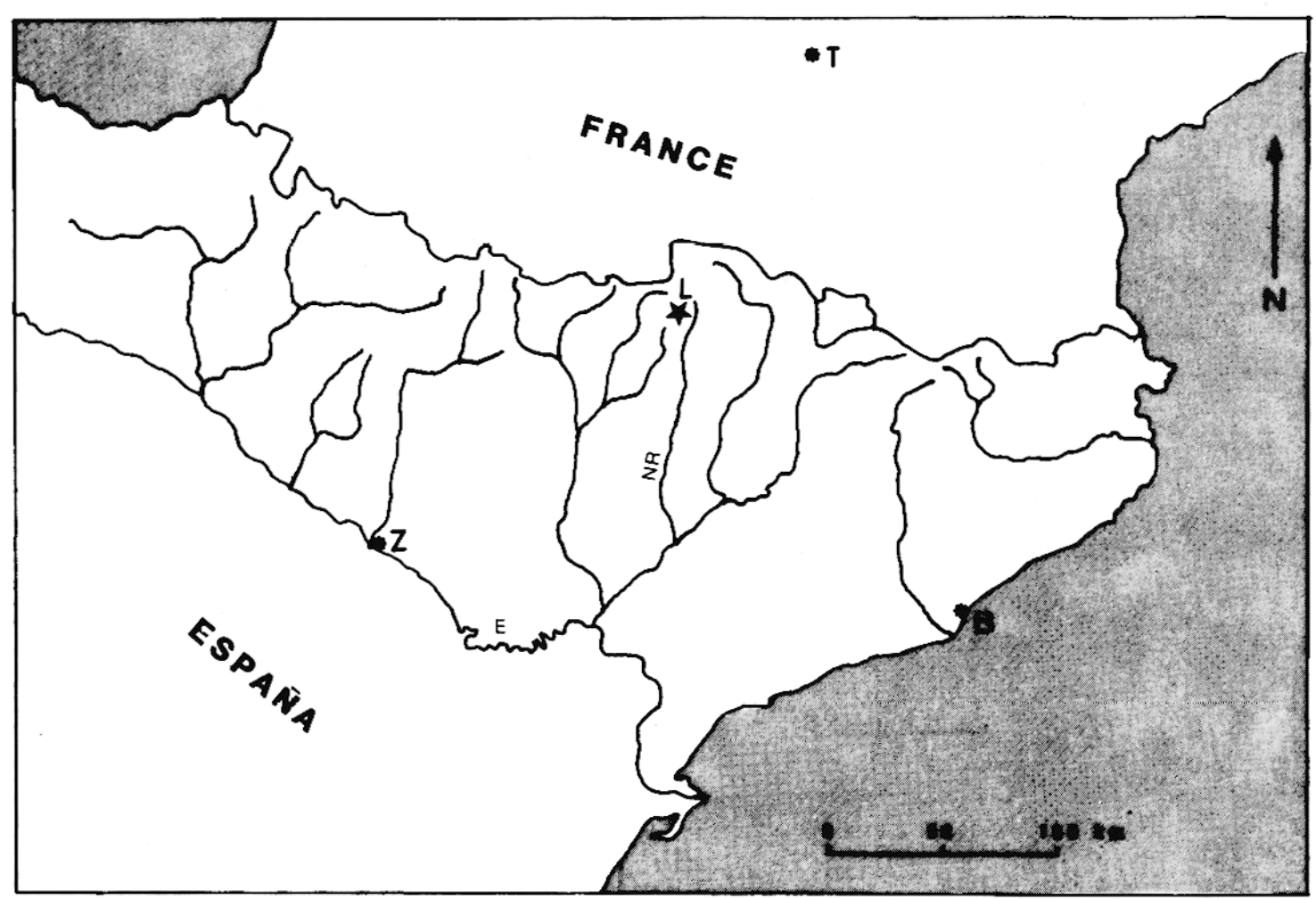

Fig. 1. - Situation du paleolac de Llestul (Bassin de la Noguera Ribagorçana). B, Barcelona; T, Toulouse; Z, Zaragoza; E, Ebro; NR, Noguera Ribagorçana.

Fig. 1. - Location of the paleolake Llestui (Noguera Ribagorçana Basin). B, Barcelona; T, Toulouse; Z, Zaragora; E, Ebro river; NR, Noguera Ribagorçana river.

\section{MÉTHODES D'ETUDE}

A Llestui, l'incision postglaciaire des torrents a favorisé la mise à jour d'une grande coupe de sédiments glaciaires, glaciolacustres et fluviotorrentiels (ice-lateral till complex) contemporains du dernier cycle glaciaire. Sur ces affleurements, des profils stratigraphiques ont été obtenus (fig. 2). Nous avons réalisé l'analyse sédimentologique des différents lithofaciès : diamictons, graviers, sables et fines. Dans tout les cas, nous avons considéré la géométrie des corps sédimentaires (relations verticales et horizontales), le type de contact entre les différents lithofaciès et les structures sédimentaires. Les résultats ont été présentés graphiquement sous forme de profils stratigraphiques verticaux, complétés avec le code de faciès proposé par Eyles et al. (1983) et Eyles and Miall (1984) (fig. 3 et 4).

\section{RESULTATS}

Les lithofaciès et les structures sédimentaires sont caractéristiques des mécanismes de sédimentation et, en conséquence, elles nous fournissent des informa- tions sur la dynamique de l'environnement glaciolacustre.

Au paléolac de Llestui nous avons reconnu l'existence de trois ensembles principaux de mécanismes sédimentaires (fig. 5 et 6 ) :

1. Processus qui sont en relation avec les instabilités (mass movement) des versants, émergés ou submergés (fig. 5). Les plus importants sont ceux produits par des mouvements de masse de la moraine latérale, avec formation de flow-tills. Le lithofaciès caractéristique de ces dépôts est un diamicton très riche en matrice argileuse et massif (code de faciès Dmm, voir fig. 3 et 4 ).

2. Processus qui sont en relation directe avec les apports des eaux de fonte supra-glaciaires et sousglaciaires (Ashley, 1988) et des eaux de ruissellement d'origine non glaciaire (fig. 5). Ces processus produisent la sédimentation des graviers et des sables dans les zones proximales, et des argiles laminées dans les zones distales (centre de la cuvette). Dans ces types de lacs, les faciès laminés correspondent parfois à des turbidites (Sturm and Matter, 1978) (fig. 3). Le régime hydrique torrentiel du bassin versant qui alimente le lac est le principal responsable de son remplissage sédimentaire. Deux grands cônes de déjection progradent vers l'intérieur de la cuvette (voir coupe de la fig. 2 et fig. 5). En conséquence, 

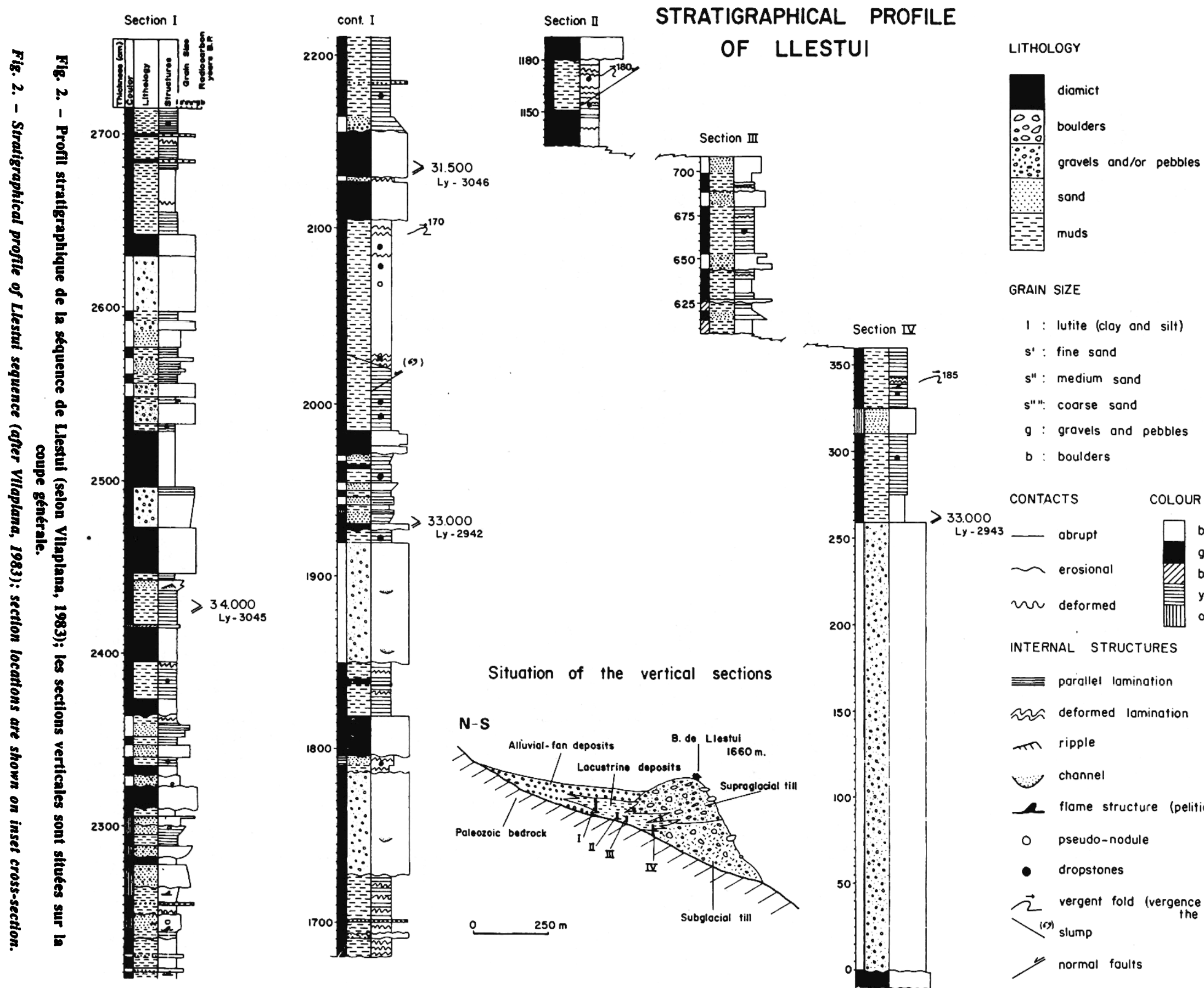

GRAIN SIZE

1 : lutite (clay and silt)

$\mathbf{s}^{\prime}$ : fine sand

$s^{\prime \prime}$ : medium sand

s" ": coorse sand

$g$ : gravels and pebbles

b : boulders

CONTACTS COLOUR

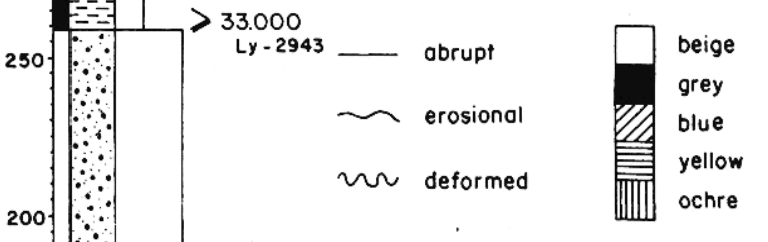

INTERNAL STRUCTURES

parallel lamination

$\approx \approx$ deformed lomination

in ripple

I. channel

a flame structure (pelitic injection)

- pseudo-nodule

- dropstones

$\overrightarrow{2}$ vergent fold (vergence direction from

(c)s slump

y normal foults 


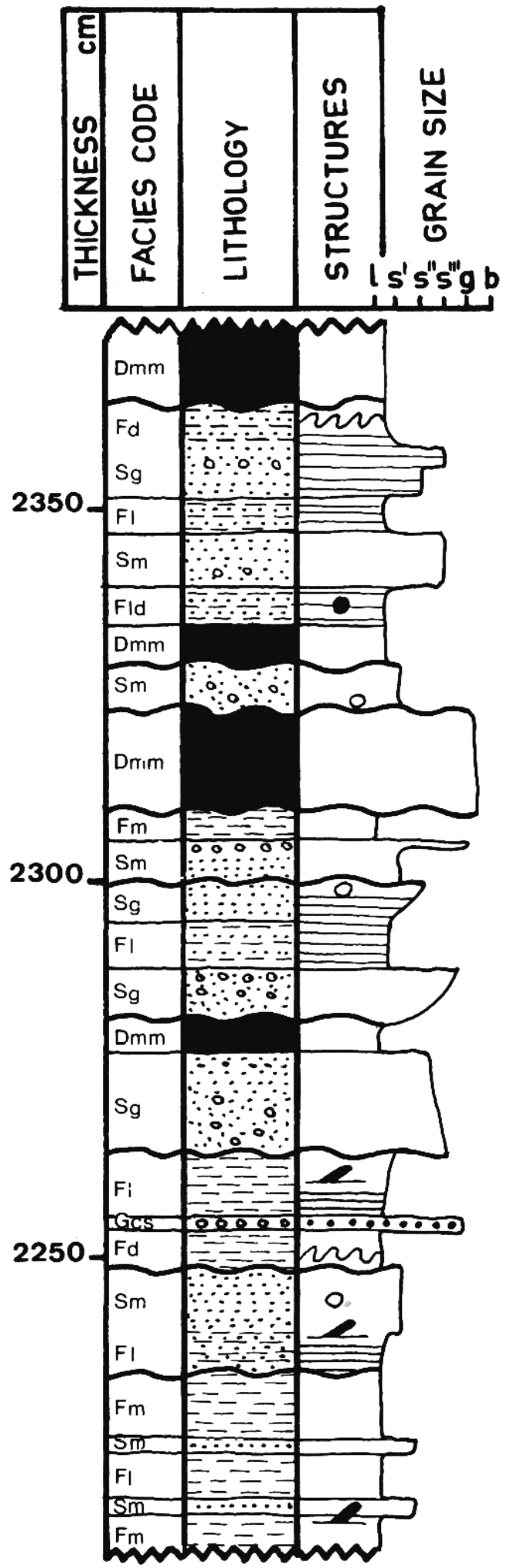

Fig. 3. - Détail de la section I (ng. 2) qui nous montre différents contacts érosifs et une couche de «dropstones». Pour la légende voir ngure 2.

Fig. 3. - Detail of section I (fig. 2) showing several erosional contacts in the sequence and a layer of dropstones. See figure 2 for legend.

Facies code : Dmm : Diamicton, matrix supported, massive. Gcs : Gravels, clast supported, stratified.

$S g$ : Sands, gradded.

Sm : Sands, massive.

$F l:$ Fines, laminated.

$F d$ : Fines, deformed lamination

Fm : Fines massive

Fld : Fines, laminated, dropstones.
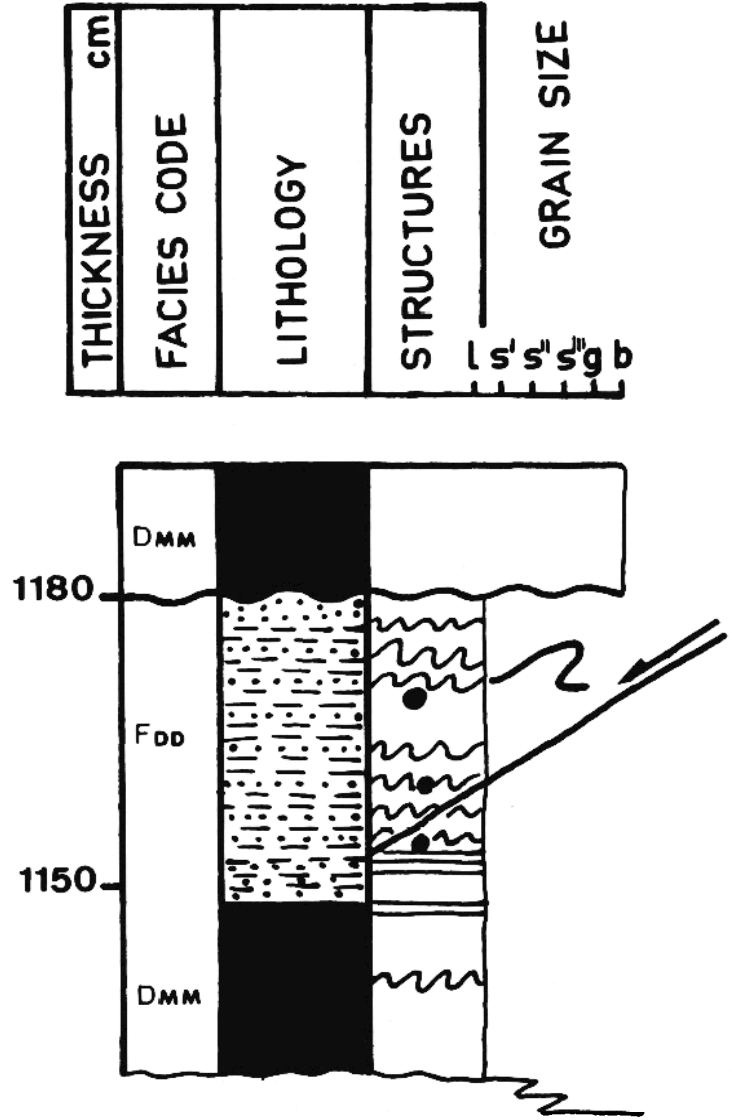

Fig. 4. - Section II (figure 2). Niveaux de rythmites déformés et faillés au-dessous des diamictons. Pour la légende voir figure 2.

Fig. 4. - Section II (figure 2). Deformed and faulted rhythmites below a diamicton. See figure 2 for legend. Facies code. DMM : Diamicton, matrix supported, massive. Fdd : Fines, deformed lamination, dropstones.

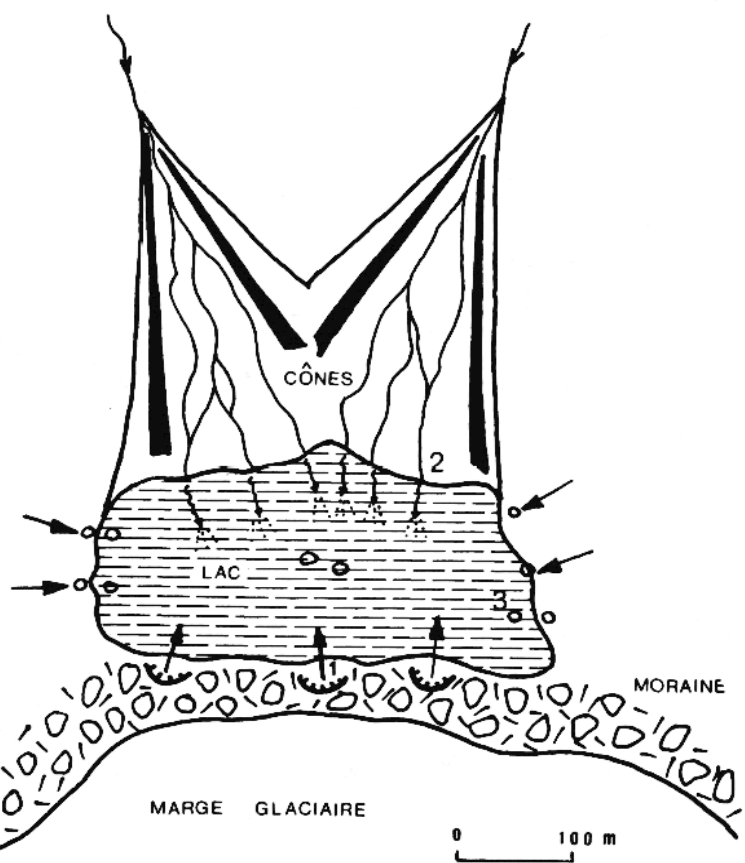

Fig. 5. - Reconstruction schématique du lac de Llestui, avec indication des principales sources de sédiment. Les numéros qui désignent les ensembles de mécanismes sédimentaires $(1,2,3)$ dans le chapitre des résultats, sont utilises sur cette figure dans le méme sens.

Fig. 5. - Schematic reconstruction of lake Llestui showing the main sedimentary sources. Numbers 1, 2, 3, indicate the sedimentary processes described in the text. 
dans les dépôts fins (F) du centre de la cuvette, on trouve des interruptions érosives de la sédimentation et des intercalations de sables et de graviers (S; G).

3. Processus qui sont en relation avec l'existence saisonnière d'une couche de glace à la surface du lac. Pendant la fonte de cette surface englacée, des chutes de pierres se produisent à l'intérieur de la cuvette. C'est la sédimentation des «dropstones» que nous trouvons ou bien isolés dans les laminites argileuses, ou bien disposés en bancs interstratifiés dans les argiles.

En résumé, on peut dire que la distribution des faciès qui résultent de ces processus est totalement conditionnée par la géométrie et par la taille de la cuvette lacustre. Sur la marge du lac, on trouve près de la moraine latérale une prépondérance de diamictons (interprétés comme flow tills et waterlain tills; Dreimanis, 1988) et près des émissaires du versant des graviers et des sables fluvioglaciaires ou fluviodeltaïques. Au centre de la cuvette de Llestui, on trouve fréquemment plusieurs intercalations des lithofaciès grossiers en contact érosif avec les faciès fins (argiles et limons laminés à sédimentation rythmique) (fig. 2 et 6 ).
L'ensemble des mécanismes décrits et leurs résultats correspondants nous permettent d'estimer une vitesse de sédimentation relativement grande. $\mathrm{Par}$ conséquent, les $27 \mathrm{~m}$ de la série stratigraphique mesurés sur les profils verticaux doivent correspondre à un remplissage rapide.

En ce qui concerne la tranche d'eau, nous estimons que son épaisseur devait varier entre un minimum de l'ordre de quelques mètres et un maximum de deux décamètres. La cote du lac n'a pas été toujours la même parce qu'elle a été sous la dépendance de la position et de la dynamique du glacier (hauteur, progradation/retraite) qui a produit l'obturation. L'élévation de la cote du lac, conséquence d'une progradation du glacier, détermine aussi une régression du front fluvio-deltaïque (voir coupe fig. 2).

\section{CONCLUSIONS}

Classiquement, les rythmites sont considérées comme le lithofaciès caractéristique des domaines sédimentaires glaciolacustres. Dans les petits lacs de marge glaciaire du type de Llestui, où les variations

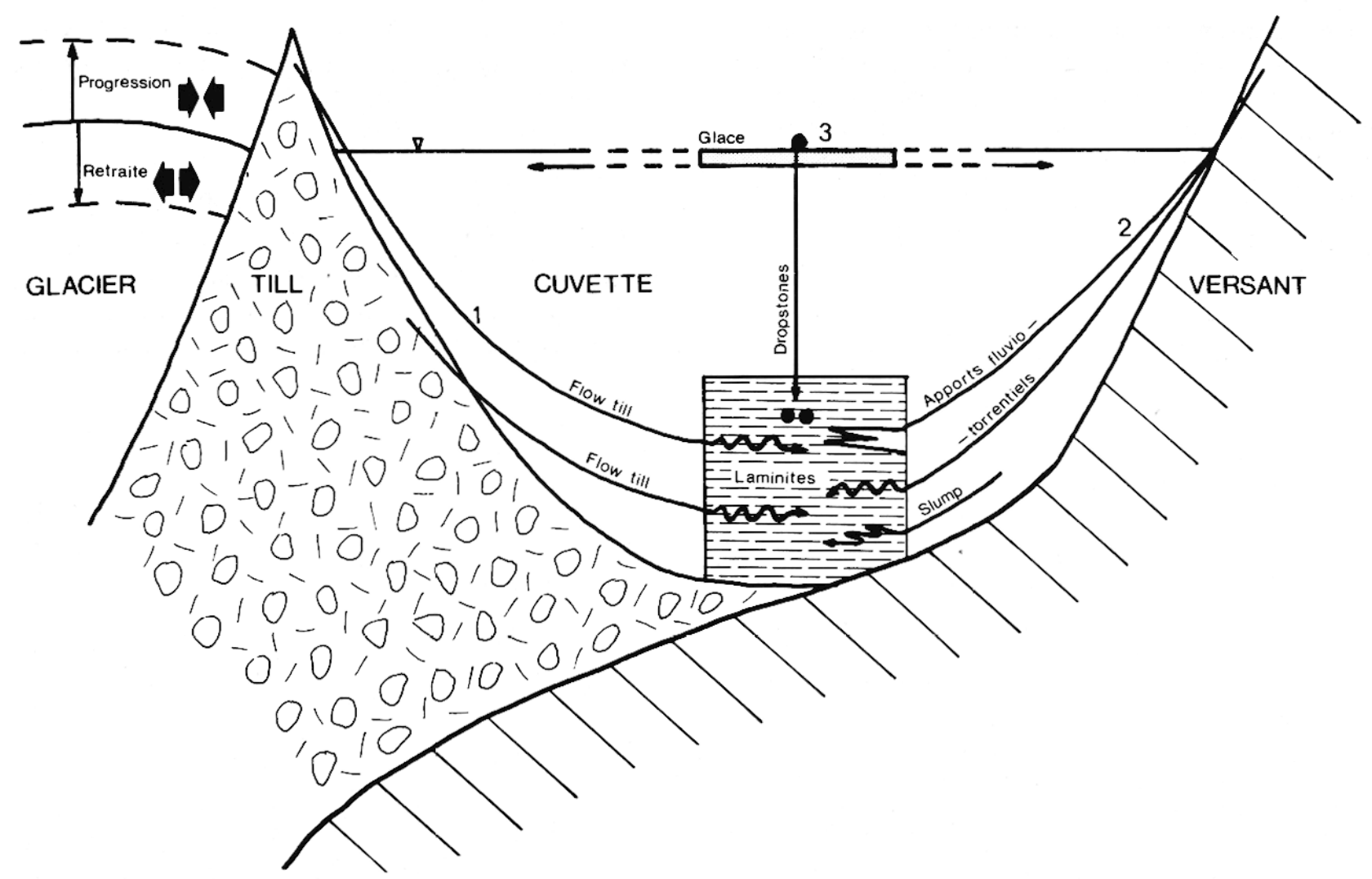

Fig. 6. - Modele schématique (sans échelle) de la sédimentation rythmique dans un petit lac juxtaglaciaire où l'on peut apprécier les variations verticales et latérales des lithofacies. La sédimentation des argiles laminées est souvent interrompue par d'autres dépóts qui s'intercalent et produisent des contacts érosifs. Les numéros qui désignent les ensembles de mécanismes sédimentaires $(1,2,3)$ dans le chapitre des résultats, sont utilisés sur cette figure dans le méme sens.

Fig. 6. - Schematic model (not to scale) of rhythmic sedimentation in a small ice-lateral lake interrupted by common erosional and interbedded contacts. We can see the vertical and lateral variations of the lithofacies. Numbers 1, 2, 3 indicate the sedimenta. ry processes described in the text. 
de la dynamique sédimentaire sont très importantes, dans le temps et dans l'espace, le dépôt des éléments fins subit de nombreuses interruptions érosives (fig. 3 et 4). En conséquence, le résultat de la sédimentation n'est pas une longue séquence d'argiles laminées mais une alternance des différents lithofaciès existants (Codes D-G-S-F).

L'exemple du paléolac de Llestui nous permet raisonnablement de supposer que les sédiments lacustres dans les lacs d'obturation latérale des glaciers quaternaires pyrénéens, sont constitués par un ensemble de lithofaciès qui présentent des relations verticales et horizontales complexes (fig. 6). Cette complexité est le résultat de la superposition des contrôles sédimentaires suivants :

- Proximité du glacier (ice-contact).

- Géométrie et taille de la cuvette (profondeur métrique à décamétrique, diamètre hectométrique).

- Localisation et typologie des sources de sédiment (Moraine, cône torrentiel).

- Dynamique des versants de la cuvette (Mass movement). tones»).

- Surface du lac englacé (production de «drops-

Nous pensons que ces observations faites à Llestui peuvent être utiles pour l'analyse sédimentologique de tous les petits paléolacs de marge glaciaire en montagne, en particulier pour ceux qui ne présentent pas de coupes et doivent être étudiés à partir de carottes.

\section{REFERENCES}

Ashley G.M., 1988. - Classification of glaciolacustrine sediments. In Goldthwait and Matsch (Eds.) : Genetic classification of glacigenic deposits, A.A. Balkema, p. 243-260.

Dreimanis A., 1988. Tills : Their genetic terminology and classification. In Goldthwait and Matsch (Eds) : Genetic classification of glacigenic deposits, A.A. Balkema, p. 17-83.

Eyles N., Eyles C.H. and Miall A.D., 1983. - Lithofacies types and vertical profile models; an alternative approach to the description and environmental interpretation of glacial diamict and diamictite sequences. Sedimentology, 30, p. 393-410.

Eyles N., and Miall A.D., 1984. - Glacial facies. In Walker, R.G. (Ed) : Facies models, Geological Assoc. of Canada. p. 15-38.

Serrat D., Vilaplana J.M. and Marti C., 1983. Some depositional models of glaciolacustrine environment in Southern Pyrenees. In Evenson et al. (Eds) : Tills and Related Deposits, A.A. Balkema, p. 132-144.

Sturm M. and Matter A., 1978. - Turbidites and varves in Lake Brienz (Switzerland) : deposition of clastic detritus by density currents. Spec. Public. Int. Ass. Sed., no 2, p. 147-168.

Vilaplana J.M., 1983 (a). - Sobre la geomorfologia de la vall de Llauset : Pirineu Ribargorçà. Rev. Investigacions Geol., $\mathrm{n}^{\circ}$ 36, p. 97-120.

Vilaplana J.M., 1983 (b). - Quaternary glacial geology of Alta Ribagorça basin (Central Southern Pyrenees), Acta Geoldgica Hispdnica, 18, $n^{\circ} 3-4$, p. 217-233. 